\title{
A Multi-Scale Line Feature Detection Using Second Order Semi-Gaussian Filters
}

\author{
Baptiste Magnier, Ghulam-Sakhi Shokouh, Binbin Xu, and \\ Philippe Montesinos
}

\begin{abstract}
EuroMov Digital Health in Motion, Univ. Montpellier, IMT Mines Alès, Alès, France baptiste.magnier@mines-ales.fr
\end{abstract}

\begin{abstract}
Among the common image structures, line feature is the extensively used geometric structure for various image processing applications, including the analysis of biomedical image with blood vessels highlighting, graph-shape structures, cracks detection, satellite images or remote sensing data. Multi-scale processing of line feature is essentially required for the extraction of more relevant information or line structures of heterogeneous widths. In this paper, a multi-scale filtering-based line detection approach using second-order semi-Gaussian anisotropic kernel is proposed. Meanwhile, a strategy is introduced to calculate the strength of the observed line feature across the different scales. The proposed technique is evaluated on real images by using their tied hand-labeled images. Finally, the experimental results and comparison of images containing different line feature widths with state-of-the-art techniques have sufficiently supported the effectiveness of our technique.
\end{abstract}

Keywords: Line-feature detection $\cdot$ Multi-scale $\cdot$ Semi-filters $\cdot$ Steerable

\section{Introduction}

Line features represent ridges and valleys in a digital image, they correspond to thin, elongated structures and ridges refer to the valleys of the inverted relief on the image surface, as illustrated in Fig. 1(b). Line feature characterization is the initial step in all aforementioned applications. Amongst the low level image structures, such as, texture, edge, corner or junction, line features is the widely applied structure in the image processing literature. Line structures on multiscale like ridges or valleys contain determinative information required in image analysis problems, such as, scene understanding, photogrammetry, biomedical [12] and remote sensing data. It is important to have a reliable line detector, especially adapted to different scales. Today, there are many post-processing methods to align segments, group or recognize shapes. These methods are all more effective when the line detector is reliable.

Two parallel step edges construct a line structure, they can be roughly extracted by the Laplacian operator, as in Fig. 1(c). Technically the step or ramp edges correspond to local maxima of the first order derivative [2], while ridges 


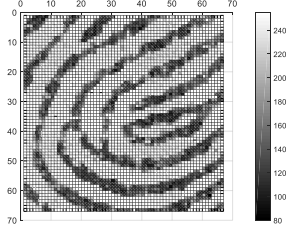

(a) Gray level image of size $67 \times 67$ coded on 8 bits.

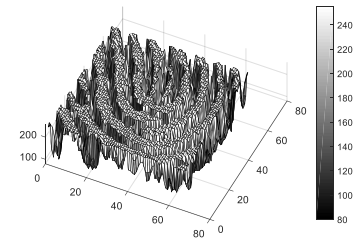

(b) Image surface of (a), ridges are at the top whereas valleys are at the bottom of the surface.

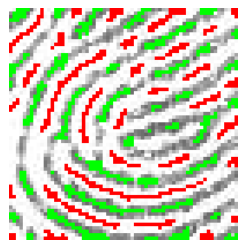

(c) Ridges/valleys extracted by Laplacian of Gaussian.

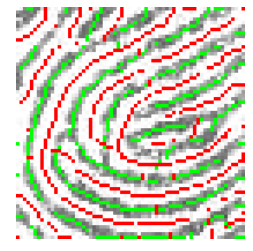

(d) Ridges/valleys extracted with the Hessian matrix.

Fig. 1. 3D representation of ridges/valleys and their extractions using the Laplacian of Gaussian and Hessian matrix [14], $\sigma=2.88$, see [13]. In (c)-(d), extracted ridges in red and valleys in green are superimposed on the original image.

are tied to local maxima of second order derivative in the local analysis of Hessian matrices of an image [5] [14]. There exist other techniques to extract line structures, including oriented filters, as presented in the next section.

In this paper, a multi-scale Second Derivative of Semi-Gaussian (SDSG) filtering technique is proposed. The line feature profiles can vary across scale space; they are detected with different filter parameters, whose outputs would be merged latter to create a single edge map. Hence, a function is proposed for the scale fusion considering the desired scale.

\section{Multi-Scale Ridge Extraction: Related Works}

Gaussian kernels as well as their derivatives are the widely employed filtering techniques for the processing of low level image structures due to their isotropy, steerability and decomposability properties. The zeroth order Gaussian kernels are used for smoothing and regularization. This section covers the main theoretical principles of multi-scale line-feature extraction in digital images.

\subsection{Isotropic filters}

For the line-structure detection, several works are based on the eigen-decomposition of the Hessian computed at each image pixel [5][13][14]. The combination of the eigen-values measures the overall strength of the ridge or the valley, as illustrated in Figs. 1(d) and 2(b). In scale space, theoretically, a pioneer work proposed by Lindeberg assumes that a ridge point is defined as a location for which the intensity assumes a local maximum (or minimum for the valleys) in the main principal curvature direction [5]. Considering an image $I_{\sigma}$ smoothed by a Gaussian of standard deviation $\sigma$, the line-structure measure of the original image $I$ is given by:

$$
\mathcal{N}_{\gamma}(I)=\sigma^{2 \gamma} \cdot\left(\left(I_{\sigma, x x}-I_{\sigma, y y}\right)^{2}+4 \cdot I_{\sigma, x y}\right),
$$

where $I_{\sigma, x x}$ and $I_{\sigma, y y}$ represent the $x$ and $y$ derivatives of the image $I_{\sigma}$ respectively, and $\gamma>0$ is termed as the scale normalization factor.

Bae et al. [1] extended the $\gamma$-normalized multi-scale Hessian matrix of Eq. 1 to derive a width-invariant and contrast-proportional second derivative magnitude map. Then a high-level processing is performed for segment formation. 


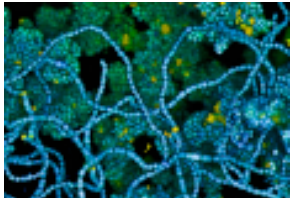

(a) Image

$116 \times 81$

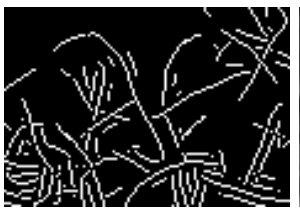

(e) SOAGK, $\Delta \theta=5^{\circ}$

$\sigma_{u}=1.81, \sigma_{v}=5 \sigma_{u}$

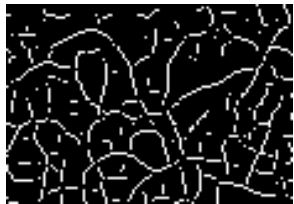

(b) Highest value of

Hessian matrix [14]

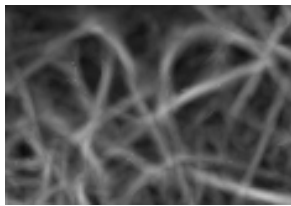

(f) Linemap of SDSG,

$\sigma_{u}=1.81, \sigma_{v}=5 \sigma_{u}$

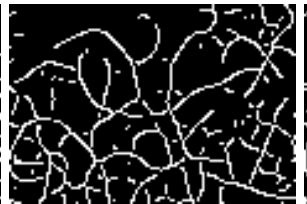

(c) Steerable filter

of order $2\left(S F_{2}\right)$ [3]

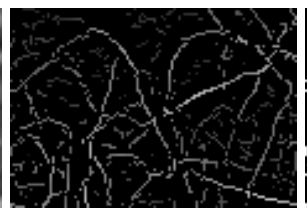

(g) Non-maxima

suppression of (f)

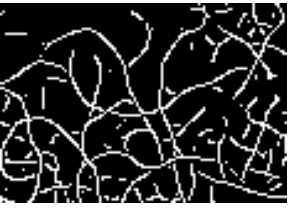

(d) Steerable filter of order $4\left(\mathrm{SF}_{4}\right)[4]$

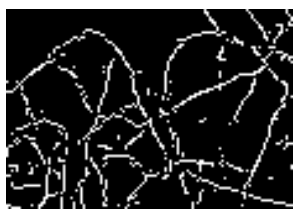

(h) Thresholding of image in $(\mathrm{g})$

Fig. 2. Extraction of ridges corresponding of long chains of streptococcus pyogenes infecting grape-like clusters of MRSA biofilm: comparison of mono-scale ridges extractors. Original image source: https://www.nikonsmallworld.com

\subsection{Oriented Filters for Line Feature Detection}

The well-known and popular steerable filters [3][4] are built by linear combination of the direct rotation of the derivatives of the basic isotropic Gaussian. Thereafter, it captures the line structure energy in the direction of the maximum response of the filter. Edge detection techniques using elongated kernels are efficient to correctly detect large linear structures [11][4][6]. The robustness against noise depends strongly on the smoothing parameters of the filter, i.e., the parameter of the filter elongation. Moreover, the elongated filters enable us to capture discontinuous line features, as illustrated in Figs. 2(d)-(e). To extract ridges, the Second-Order Anisotropic Gaussian Kernel (SOAGK) can be applied [6]. Considering the vertical anisotropic Gaussian directed at $\theta=0^{\circ}$, its second derivative in the $x$ direction is:

$$
\mathcal{G}_{\sigma_{u}, \sigma_{v}, \theta=0}^{\prime \prime}(x, y)=\frac{x^{2}-\sigma_{u}^{3}}{2 \pi \sigma_{u}^{5} \sigma_{v}} \cdot \mathrm{e}^{-\frac{1}{2}\left(\frac{x^{2}}{\sigma_{u}^{2}}+\frac{y^{2}}{\sigma_{v}^{2}}\right)} .
$$

The choice of $\sigma_{v}>\sigma_{u}$ enables to build a narrow filter smoothing mostly in the $y$ direction while enhancing valleys in the $x$ direction. Now, this $2 \mathrm{D}$ kernel can be oriented in different directions to capture line structures in the image, see Fig. 2(e). To this end, this anisotropic choice produces a smoothing alongside the ridge/valley, which helps to extract easily elongated features, even disturbed by noise. On the contrary, kernels having parameters $\sigma_{v} \approx \sigma_{u}$ are equivalent to a Steerable Filter of order $2\left(S F_{2}\right)[3]$ and may highlight undesirable features as noise which are interpreted as small, non-elongated ridges [6]. Finally, SOAGK at different scales are applied in [6] to detect the line structures and the combination is done by means of the maximum among the different obtained line-maps. 


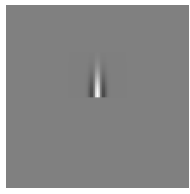

(a) $\theta=0^{\circ}$

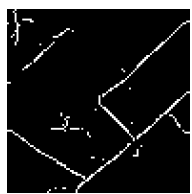

(f) $\Delta \theta=2^{\circ}$

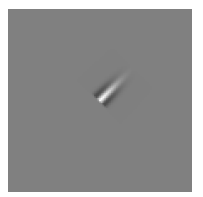

(b) $\theta=45^{\circ}$

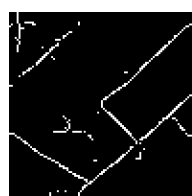

(g) $\Delta \theta=5^{\circ}$

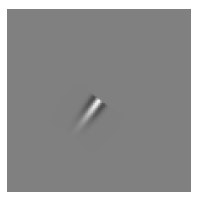

(c) $\theta=217^{\circ}$

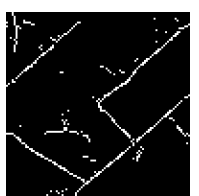

(h) $\Delta \theta=10^{\circ}$

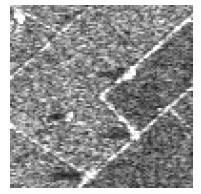

(d) Image $96 \times 96$

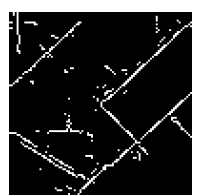

(i) $\Delta \theta=22.5^{\circ}$

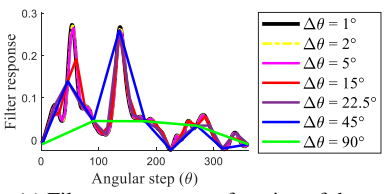

(e) Filter response as a function of the angular sampling for the central pixel in (d)

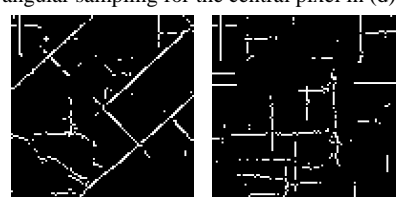

(j) $\Delta \theta=45^{\circ}$

(k) $\Delta \theta=90^{\circ}$

Fig. 3. Ridge detection as a function of the angular step $\Delta \theta$, with $\sigma_{d}=1.8, \sigma_{s}=5 \sigma_{d}$, and SDSG responses (clockwise) at a pixel tied to a bended ridge.

\section{Second-Derivative of a Semi-Gaussian Filter (SDSG)}

The basic idea of the developed filter is to consider paths (i.e., ridges or valleys) crossing each pixel. Inspired by [10], the proposed technique Second-Derivative of a Semi-Gaussian Filter (SDSG) represents a truncated 2nd derivative of an anisotropic Gaussian which can be steered. It's able to detect bended ridges due to two elongated and oriented filters in two different directions.

\subsection{Concept of the SDSG}

The main idea of SDSG is to "cut" the second order anisotropic Gaussian kernel (Eq. 2) using a Heaviside function and, then, steer this filter in all directions around the considered pixel: from 0 to $360^{\circ}$. Hence, the SDSG can be built by combining a vertical semi-Gaussian on the one hand and its horizontal second derivative on the other hand. Mathematically, it is defined by:

1. a semi-Gaussian for the smoothing in the $y$ direction (vertically):

$$
\mathcal{G}\left(\sigma_{s}, t\right)=H(t) \cdot \mathbf{e}^{\frac{-t^{2}}{2 \cdot \sigma_{s}^{2}}} \text {, with } \sigma_{s} \in \mathbb{R}_{+}^{*}, t \in \mathbb{R} \text { and } H \text { the Heaviside function, }
$$

2. a second derivative of a Gaussian in the $x$ direction (horizontally):

$$
\mathcal{G}^{\prime \prime}\left(\sigma_{d}, t\right)=\frac{t^{2}-\sigma_{d}^{2}}{\sigma_{d}^{4}} \cdot \mathbf{e}^{\frac{-t^{2}}{2 \cdot \sigma_{d}^{2}}} \text {, with } \sigma_{d} \in \mathbb{R}_{+}^{*} \text { and } t \in \mathbb{R} .
$$

For signal and image processing, $t$ represents an integer. The Fig. 3(a) shows an example of SDSG, constructed with these two functions, respectively, $\mathcal{G}$ at the vertical and $\mathcal{G}^{\prime \prime}$ at the horizontal. In order to create an anisotropic (elongated) filter, the support of the smoothing half-filter must be greater than the support of the filter containing the derivative, that is to say $\sigma_{s}>\sigma_{d}$. Then, to obtain a rotated version of the SDSG, this filter is applied in several directions $\theta$ from 0 to $360^{\circ}$. The original rotation is centered on the middle of the basis filter; for a better understanding, the rotation center corresponds to the middle of the image in Fig. 3(a), and the SDSG is rotated from this point in Figs. 3(b) 
and (c). Thereafter, the image convolution with the steered filters allows to compute a derivative information at each desired direction (as shown in Fig. $3(\mathrm{e})$ ). Then, the line structure strength $\mathcal{L}$ is calculated using a local directional maximization/minimization:

- addition of the two local maxima regarding ridge detection,

- addition of the two local minima regarding valley detection.

The direction perpendicular to the line structure, called $\vec{\eta}$ is calculated by the bisector between these two local directions (maxima or minima). Then, the line structures can be extracted with non-maxima suppression (NMS) process by deleting local non-maxima in the $\vec{\eta}$ direction, same strategy as in [2]. The Fig. 3 (e) illustrates two local maxima tied to the directions of a bended line (here the $\vec{\eta}$ direction is around $90^{\circ}$ direction). When the angular step $\Delta \theta$ is well discretized, such a filtering technique allows computing two precise directions of the line structure. The two directions cannot be correctly calculated when the angular step is too spaced $\left(\Delta \theta>5^{\circ}\right.$, Figs. $\left.3(\mathrm{f})-(\mathrm{k})\right)$.

In [9], an anisotropic directional filter is implemented considering difference of two half rotating Gaussian filters (DoG mechanism), which can approximate the SDSG filter. Meanwhile, a multi-scale approach has been developed by selecting the maximum response among the scales [9]. Such a normalization function may miss some thin objects, as illustrated in Fig. 5(c)-(d) and next subsection.

\subsection{Scale fusion of the SDSG}

In one dimension, the $\sigma$ of the Gaussian derivatives depends on the line width of the structures to be detected, as shown in Fig. 4(a)-(b). The SDSG corresponds to a semi-filter. It can be seen as a scan of the projected pixels in all the directions around the considered pixel, illustrated by the signals in Fig. 3(e). When the SDSG is steered in the line feature direction, the $\sigma_{s}$ parameter allows an elongated smoothing in the line direction, whereas the $\sigma_{d}$ (tied to the 2 nd derivative which is perpendicular to the line) captures the line structure strength. For multi-scale line structure detection, the maximum value among the different filter responses can be selected [9], as in Fig. 4(b). However, it may not be sufficient, especially in real conditions. Consequently, we propose the following improved scale function:

$$
\mathcal{F}_{\sigma}(\mathcal{L})=\left(\sigma^{\frac{1}{\sigma}}+\frac{1}{\sqrt{\sigma}}\right) \cdot \mathcal{L},
$$

where $\mathcal{L}$ represents the filtered image line structure at scale $\sigma$.

This function allows to improve line structure enhancement at the corresponding scale. Its values are always superior than 1, which is efficient for large scales. Thin line features are also well highlighted, as illustrated in Fig. 4(c). Finally, the $\sigma_{d}$ parameter is considered in the Eq. 3, regarding the SDSG filter, see Fig. 5(h) where thin and large elongated structures are better connected. 


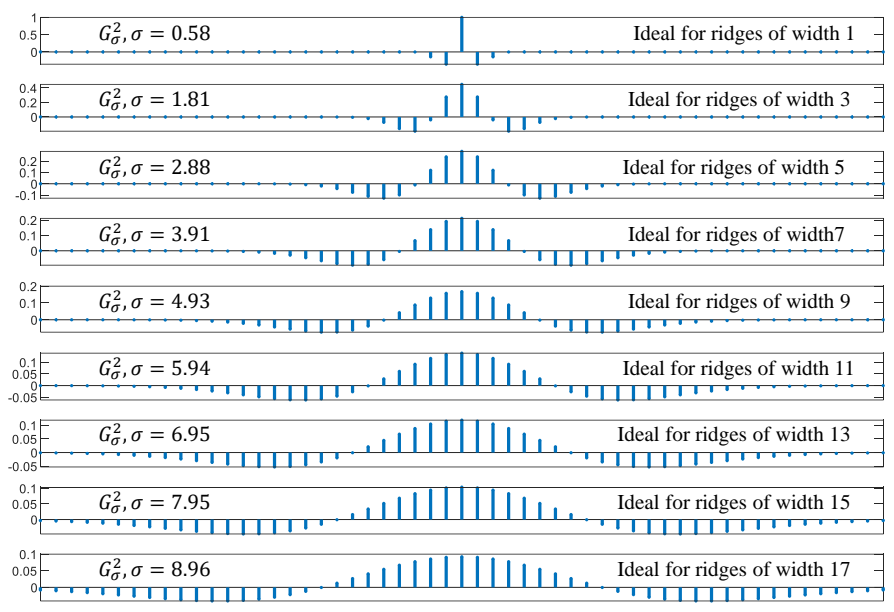

(a) Discrete second derivatives of the Gaussian with different parameters tied to the ridge width

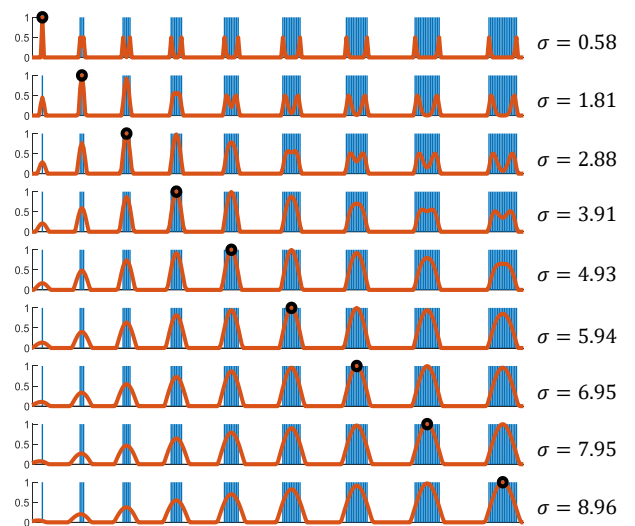

(b) Convolution of the signal with the second derivatives of the Gaussian on the left (without normalization)

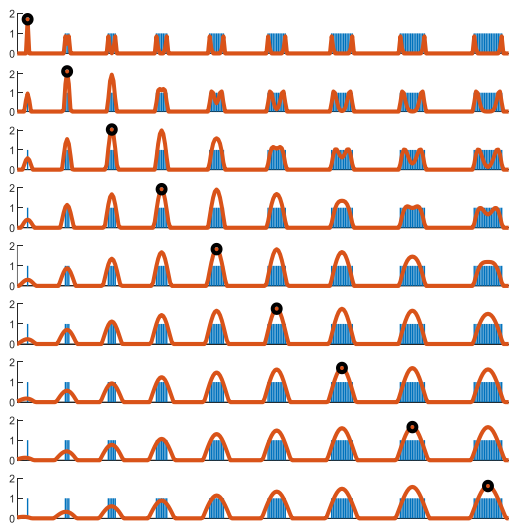

(c) Convolution of the signal with the second derivatives of the Gaussian on the left (with normalization)

Fig. 4. Ridge highlighting in one dimension (1D) by convolution with different second derivative of Gaussians in (a), detailed in [13]. In (b), ridges are highlighted with the different Gaussian convolutions and (c) takes into account Eq. 3. The original signal containing separated ridges of growing widths: $1,3,5 \ldots .17$ is displayed by the blue bars in (b)-(c) while the convolved signals are plotted in orange and the maximum of the signal is displayed by the black circles for each scale $\sigma$, exhibited between (b) and (c).

The fusion procedure of the multi-scale SDSG can be summarized as follows: (i) Filtering the image with each possible SDSG at different directions $\theta$ and scales (but with same ratio $\frac{\sigma_{s}}{\sigma_{d}}$ ), then compute the line strength $\mathcal{L}$.

(ii) Retaining the strongest response $\mathcal{L}$ after applying the Eq. 3 and its tied direction $\vec{\eta}$ for each pixel.

(iii) Suppress non-maxima pixels in the $\vec{\eta}$ direction for the fused image.

The next section presents evaluations and results on real images. 


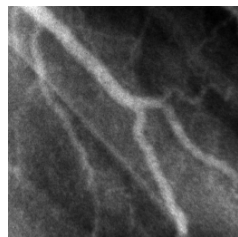

(a) Original image $256 \times 256$

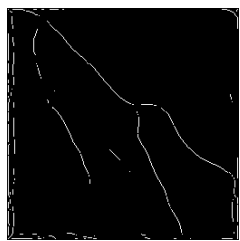

(f) Multi-scale result [1]

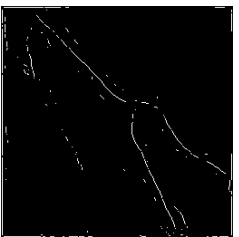

(b) Lindeberg result [5]

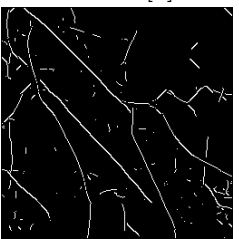

(g) Multi-scale SOAGK result [6]

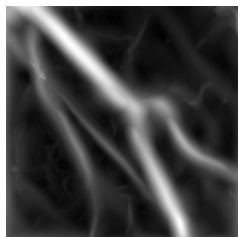

(c) SDSG fusion, max function [9]

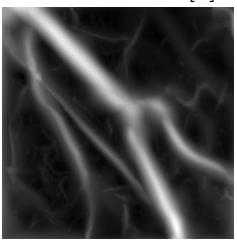

(h) SDSG fusion, with Eq. 3

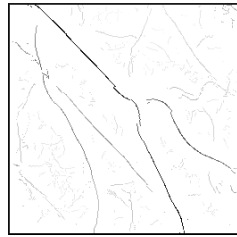

(d) Non-maxima suppression of (c)

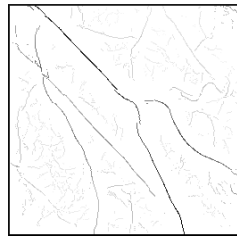

(i) Non-maxima suppression of $(\mathrm{h})$

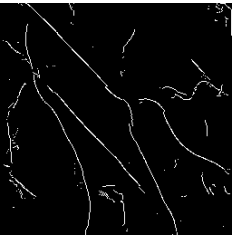

(e) Thresholding of $(\mathrm{d})$

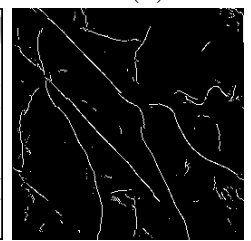

(j) Thresholding of (i)

Fig. 5. Blood vessel extraction of different widths by non-maxima suppression (NMS) and thresholding in a Magnetic Resonance Angiography image. In (b), (f) and (g), 20\% of the highest pixels are preserved while $30 \%$ are preserved for (e) and (j). Note that (d) and (i) are inverted images. Here, scales varied between 1.81 and 6.95, see [13].

\section{Experimental results and evaluation}

Experiments are carried out on real images. First, a dataset containing fungi images with manually annotated ground truth $G_{t}$ is used [6]. To evaluate the line feature detection, the Normalized Figure of Merit method [7] is employed. Let $D_{c}$ be the detected contour map of an image. Comparing pixel by pixel $G_{t}$ and $D_{c}$, a simple evaluation based on pixel-wise comparison leads to the definition of the following indicators:

- True Positive $(T P)$, common points of $G_{t}$ and $D_{c}$,

- False Positive $(F P)$, spurious detected edges of $D_{c}$,

- False Negative $(F N)$, missing boundary points of $D_{c}$,

- True Negative $(T N)$, common non-edge points.

The normalized $\mathcal{N}$ edge detection evaluation measure is, for $F N>0$ or $F P>0$ :

$$
\mathcal{N}\left(G_{t}, D_{c}\right)=\frac{1}{F P+F N} \cdot\left[\frac{F P}{\left|D_{c}\right|} \cdot \sum_{p \in D_{c}} \frac{1}{1+\delta \cdot d_{G_{t}}^{2}(p)}+\frac{F N}{\left|G_{t}\right|} \cdot \sum_{p \in G_{t}} \frac{1}{1+\kappa \cdot d_{D_{c}}^{2}(p)}\right],
$$

where $(\delta, \kappa) \in] 0,1]^{2}$ represent two scale parameters $[7],|\cdot|$ denotes the cardinality of a set, and $d_{A}(p)$ is the minimal Euclidian distance between a pixel $p$ and a set $A$. So, if there are no error, i.e., $F P=F N=0$, then $\mathcal{N}=1$. Therefore, the measure $\mathcal{N}$ calculates a standardized dissimilarity score; the closer the evaluation score is to 1 , the more the edge detection is qualified as suitable. On the contrary, a score close to 0 corresponds to a poor detection of contours.

The aim here is to get the best contour map in a supervised way. For that, the line features are extracted after a suppression of the local non-maxima, 


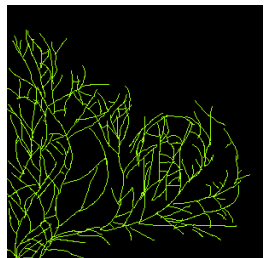

(a) Image annotated by hand (b) $F_{\alpha}$ measure, tied to $T P, F P$, and $F N$

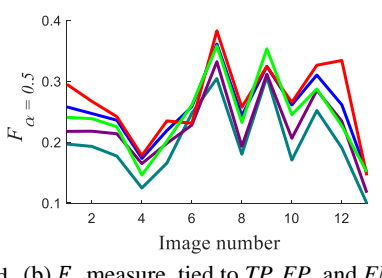

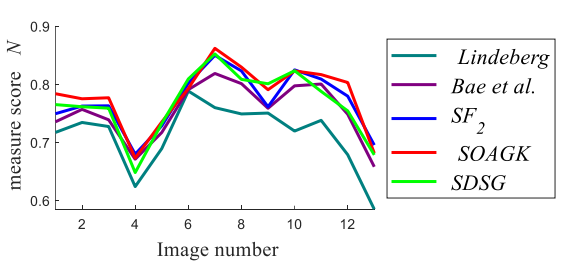

(c) Edge detection evaluation $N$

(d) Legend

Fig. 6. Evaluation of the ridge extraction technique on real images (Fungal images [6]). Detected line structures of image 3 are available in Fig. 7(a).

then a threshold by hysteresis is applied to obtain a binary segmentation [2]. Theoretically, to be objectively compared, the ideal contour map of a measure must be a $D_{c}$ at which the supervised evaluation gets the highest score [7][8]. In addition, from proper binary confusion matrix, the precision $\left(\mathrm{P}_{\text {rec }}\right)$ and recall $\left(\mathrm{R}_{\mathrm{ec}}\right)$ evaluations are computed, given the overall quality expressed in terms of the $F_{\alpha}$-measure with $\alpha=0.5$ allowing a equal penalization between $F N$ and $F P$ :

$$
F_{\alpha}=\frac{\mathrm{P}_{\mathrm{rec}} \cdot \mathrm{R}_{\mathrm{ec}}}{\alpha \mathrm{P}_{\mathrm{rec}}+(1-\alpha) \mathrm{R}_{\mathrm{ec}}} \text { with } \mathrm{P}_{\mathrm{rec}}=\frac{T P}{T P+F P} \text { and } \mathrm{R}_{\mathrm{ec}}=\frac{T P}{T P+F N},
$$

The SDSG filter is compared with 4 other multi-scale feature line detection techniques, namely: Lindeberg [5], Bae et al. [1], $S F_{2}$ [3] and SOAGK [6]. Evaluation scores are presented in Figs. 6(b)-(c) for $F_{\alpha}$ and $\mathcal{N}$ measures respectively. Usually, scores achieved by SDSG are similar to those of the SOAGK, showing the reliability of the proposed filter. Both are better than Lindeberg, $S F_{2}$ and Bae et al. which uses a post processing segment formation. Visually, detections obtained by the SDSG are close to those derived from the SOAGK, excepted that SOAGK creates many straight (small) segments for isolated points, see Fig. 7(a).

In order to interpret the output of the SDSG versus the state of the art techniques for line feature detection and extraction, the comparative tests have been carried out on different real images shown in Fig. 7, including (a) fungal, (b) cart wheel picture, (c) satellite image, (d) angiography to detect blood vessels, (e) aerial image, (f) a noisy biomedical image to detect and extract filaments.

Taking into account that the original images are noisy and blurred, the Lindeberg filtering [5] extracted the impure desired lines in spite of non-maxima suppression. The Bae et al. [1] output is better visually in extracting finer lines with higher precision because of its segment formation created by the high-level processing. Considering the steerable filter $\left(S F_{2}\right)$, it has detected more line features of varied scale. The SOAGK has rather extracted more connected line features, which is considered a strength point in filtering. The SOAGK in general demonstrates good results, particularly for elongated ridges; but too thin blood vessel as filaments and roads are not well detected, while some extracted lines are tripled and blobs are extracted as lines, penalizing this line detector. The proposed SDSG obviously has demonstrated significant result in case of noise suppression. Indeed, visually desired line features as with less or no post processing need for final output, while looking the original images. 


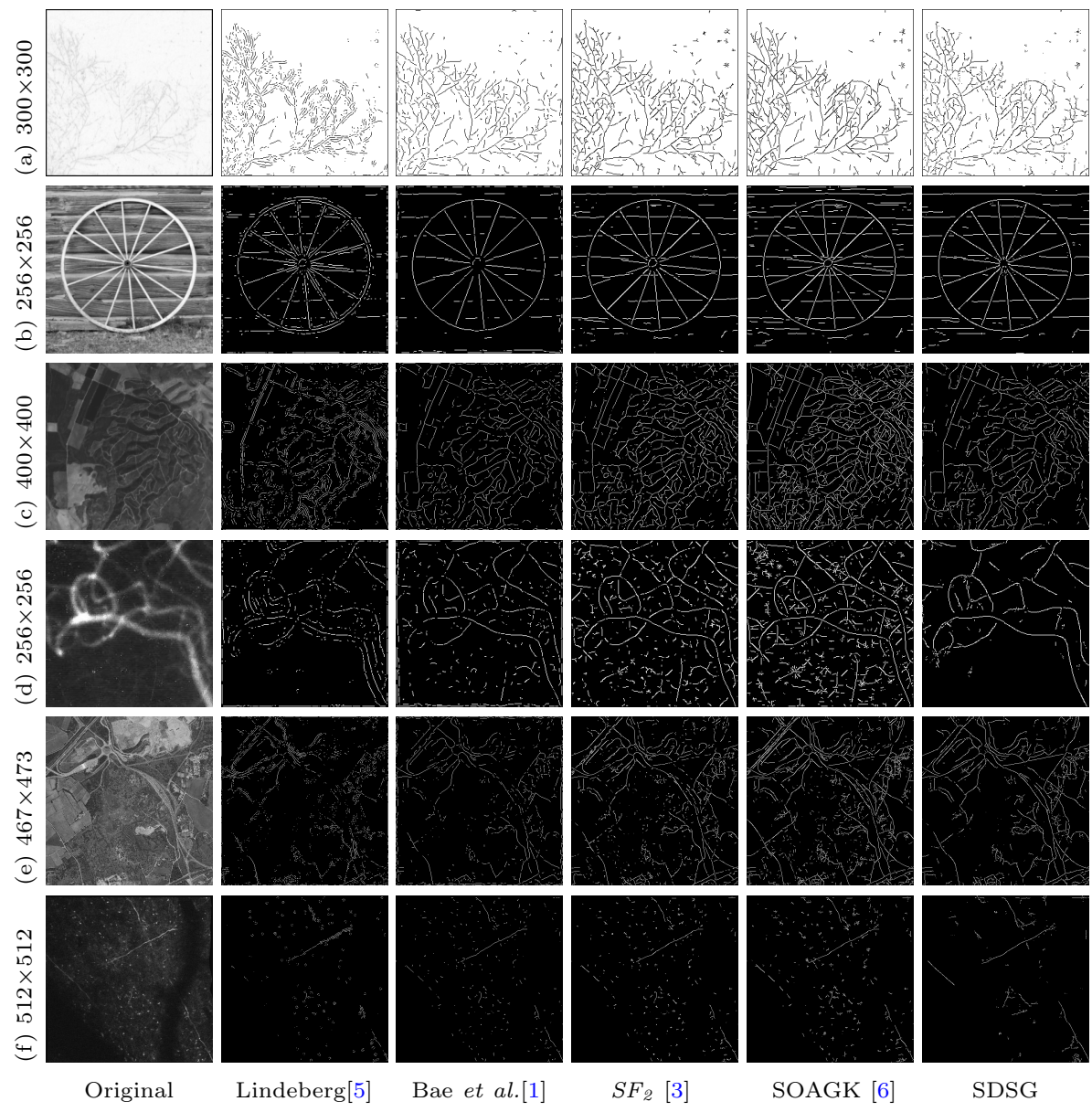

Fig. 7. Line-feature extraction on real images with multi-scale filtering methods. The images in (a) correspond to the 3rd image in Fig. 6 in the evaluation. For the images (b)-(f), detected lines correspond to the same percentage of highest pixels after NMS per method, respectively (b) $60 \%$, (c) $50 \%$, (d) $40 \%$, (e) $35 \%$, (f) $5 \%$.

The SDSG filter has extracted desirable line features in general, and in particular in Fig. 7 (c) roads (d) blood vessels, (e) large roads and (f) filament without too many undesirable false positive points. Usually, the extracted lines are more pure using the same thresholding ratio, comparing other techniques.

\section{Conclusion}

In this paper a multi-scale filtering approach for line feature detection has been proposed. The proposed approach can be adapted to noisy environments, and is also reliable to detect line feature with heterogeneous types, widths, and prominence. An optimal scale selection function for multi-scale processing is the main contribution of this approach. This approach has been compared to different 
types of multi-scale filtering methods, including isotropic (using the Hessian matrix) and oriented filters (isotropic or anisotropic). Quantitative and qualitative experiments regarding real images of different types and scales have shown the optimal efficiency and very promising results of the SDSG technique compared with the three major techniques of the state of the art.

Future work will examine the fusion of line feature detection with different ratios of $\frac{\sigma_{s}}{\sigma_{d}}$ (described in Sec. 3.1) which will add another dimension to our model. Further evaluations could involve the scales of the detected features, not only the positioning of the detection, as assessed here with $F_{\alpha}$ measure. The multi-scale responses can also serve as input layer of neural networks in biomedical applications to improve the contrast between line features and background, as explored in [12]. SDSG could bring more improvement in this type of applications.

\section{References}

1. Bae, Y., Lee, W.H., Choi, Y., Jeon, Y., Ra, J.: Automatic road extraction from remote sensing images based on a normalized second derivative map. IEEE GRSL 12(9), 1858-1862 (2015)

2. Canny, J.: A computational approach to edge detection. IEEE TPAMI pp. 679-698 (1986)

3. Freeman, W., Adelson, E.H.: The design and use of steerable filters. IEEE TPAMI 13(9), 891-906 (1991)

4. Jacob, M., Unser, M.: Design of steerable filters for feature detection using cannylike criteria. IEEE TPAMI 26(8), 1007-1019 (Aug 2004)

5. Lindeberg, T.: Edge detection and ridge detection with automatic scale selection. Int. J. of Comput. Vis. 30(2), 117-156 (1998)

6. Lopez-Molina, C., De Ulzurrun, G., Baetens, J., Van den Bulcke, J., De Baets, B.: Unsupervised ridge detection using second order anisotropic gaussian kernels. Sign. Proc. 116, 55-67 (2015)

7. Magnier, B.: Edge detection evaluation: A new normalized figure of merit. In: IEEE ICASSP. pp. 2407-2411 (2019)

8. Magnier, B., Abdulrahman, H., Montesinos, P.: A review of supervised edge detection evaluation methods and an objective comparison of filtering gradient computations using hysteresis thresholds. Journal of Imaging 4(6), 74 (2018)

9. Magnier, B., Aberkane, A., Borianne, P., Montesinos, P., Jourdan, C.: Multi-scale crest line extraction based on half gaussian kernels. In: IEEE ICASSP. pp. 51055109 (2014)

10. Montesinos, P., Magnier, B.: A new perceptual edge detector in color images. In: ACIVS. pp. 209-220. Springer Berlin Heidelberg (2010)

11. Perona, P.: Steerable-scalable kernels for edge detection and junction analysis. IVC. 10(10), 663-672 (1992)

12. Sanchez, C.F., Ivan, C.A., Arturo, H.A., Martha Alicia, H.G., Sergio Eduardo, S.M.: Automatic segmentation of coronary arteries in x-ray angiograms using multiscale analysis and artificial neural networks. Applied Sciences 9(24) (2019)

13. Shokouh, G.S., Magnier, B., Xu, B., Montesinos, P.: Ridge detection by image filtering techniques: a review and an objective analysis. Pattern Recognition and Image Analysis 31(2), to appear (2021)

14. Steger, C.: An unbiased detector of curvilinear structures. IEEE TPAMI 20(2), 113-125 (1998) 